\title{
Male biased sex ratio in the Mediterranean fruit fly Ceratitis capitata, an example of Y-chromosome meiotic drive
}

\author{
RM Shahjahan ${ }^{1}$, PA Rendon ${ }^{2}$, LM Cook ${ }^{3}$ and RJ Wood \\ Faculty of Life Sciences, University of Manchester, Stopford Building-3.614, Oxford Road, Manchester M13 9PT, UK
}

\begin{abstract}
A case of $\mathrm{Y}$-chromosome meiotic drive is reported in the Mediterranean fruit fly Ceratitis capitata. It arose in an irradiated male and results in excess of males. Male excess is inherited strictly from father to son. A Y-linked factor $M P$ (male producer) is proposed. Higher drive can be selected, but distortion declines rapidly in the absence of selection. Hybrid males from crosses between driving males and nondriving females also show drive but to a reduced extent, suggesting the action of suppressors. Sex ratio distortion is independent of postzygotic mortality, and is not associated with an obvious chromosome arrangement. Spermiogenesis in driving males is characterised by abnormalities in sperm tails and reduced numbers in some sperm cysts, whereas
\end{abstract}

neighbouring cysts of the same $M P$ testis are essentially wild type. The average number of missing sperms plus deformed sperms approximates to the average depression in female recovery among the progenies of siblings, suggesting that most of the missing or abnormal sperms would have given rise to females, that is, they would have been X-bearing. To explain the heterogeneity between neighbouring cysts, a theory is proposed that links it to variation in X-chromosome sensitivity to $M P$, arising by random suppression of the genetic basis of sensitivity during the six mitotic divisions in the origin of the cyst from its stem cell before meiosis.

Heredity (2006) 96, 464-470. doi:10.1038/sj.hdy.6800824; published online 5 April 2006

Keywords: meiotic drive; spermiogenesis; sex ratio; Y-chromosome; Ceratitis capitata

\section{Introduction}

The Mediterranean fruit fly (medfly) Ceratitis capitata (Wied.) is a serious pest of fruits in warmer parts of the world. The subject of this study is a strain showing inherited sex ratio distortion in favour of males in newly emerged adult flies. It is descended from a single medfly male exposed to X-irradiation (Wood et al, 1986). The responsible factor was named MP (male producer), and the degree of male excess associated with $M P$ was shown to increase when the temperature was reduced from 26 to $18^{\circ} \mathrm{C}$ in pupae aged $72-96 \mathrm{~h}$ (Wood and Shahjahan, 1988). This critical period of temperature sensitivity corresponds with the onset of medfly spermatogenesis, at which time male meiosis has entered prophase in the earliest primary spermatocytes to be formed (Anwar et al, 1971; Causse, 1972).

The male-determining region in C. capitata has been identified on the long arm of the Y-chromosome (Willhoeft and Franz, 1996). A male factor $M$ is believed to repress a female-determining transformer gene Cctra (Pane et al, 2002, 2005). MP male meiosis was examined

Correspondence: RJ Wood, Faculty of Life Sciences, Manchester University, Stopford Building-3.614, Oxford Road, Manchester M13 9PT, UK. E-mail: roger.wood@man.ac.uk

${ }^{1}$ Current address: Institute of Food and Radiation Biology, Atomic Energy RE, Dhaka, Bangladesh

${ }^{2}$ Current address: Moscamed Program, USDA PPQ, c/o US Embassy, Guatemala City, Guatemala.

${ }^{3}$ Current address: Manchester Museum, Manchester, UK.

Received 17 November 2005; accepted 7 March 2006; published online 5 April 2006 in feulgen-stained squash preparations by Wood et al (1986), who reported an appearance of normality. There was no sign of $\mathrm{Y}$-autosome or $\mathrm{Y}-\mathrm{X}$ exchange.

Here we describe the pattern of inheritance of MP. We also investigate whether its effect on sex ratio is associated with postzygotic mortality, and we report on cytological studies of sperm production, to investigate the possibility of a prezygotic mechanism, causing sperm loss or abnormality. As in other insect species (Phillips, 1970), the testis of C. capitata is partitioned into a series of cysts, each containing a group of spermatids (later spermatozoa) developing in synchrony within a sheath, all derived from an individual stem cell. We have observed the number of spermatids, normal and abnormal, per cyst, comparing an MP strain with two control strains (with normal sex ratios). Heterogeneity observed between cysts within a single testis is interpreted with a theory for its origin.

Ultrastructural details of normal and MP-influenced spermiogenesis are to be published separately.

\section{Materials and methods}

\section{Strains}

A425: A strain generating an excess of males owing to an inherited factor designated MP. It was founded from a single male of Hawaiian origin, exposed as 7-day puparia to $14 \mathrm{~Gy} \mathrm{X}$-rays at $1.2 \mathrm{~Gy} / \mathrm{min}$, using a PANTAK $300 \mathrm{~K}$ X-ray generator operating at $300 \mathrm{kV}, 10 \mathrm{~mA}$, crossed to a nonirradiated female. The $F_{1}$ males were pair mated to nonirradiated females, and the eight 
adults, all male, recovered from one of these back-crosses were crossed to nonirradiated females to found strain A425 (Wood et al, 1986). For two generations, it yielded around $35 \%$ females, after which the excess of males began to decline, although it could be restored to the original level by breeding from single pair progenies with most distorted sex ratios (unpublished data).

$G$ : Derived from the 37th generation of A425, being pooled progeny of four pair matings selected for $M P$ expression. These showed the highest proportion of males yet to be observed at adult emergence. Sex ratio in $\mathrm{G} \times \mathrm{G} \mathrm{F}_{1}$ (parents kept at $18^{\circ} \mathrm{C}$ degrees during days $1-5$ of puparial development) ranged from $13.9(n=36)$ to $27.3(n=84) \%$ females. Sex ratio in samples of their progeny $\left(\mathrm{G} \times \mathrm{G} \mathrm{F}_{2}\right)$ (parents kept throughout life at $26^{\circ} \mathrm{C}$ ) ranged from $28.6(n=154)$ to $36.9(n=347) \%$ females.

$H$ : Derived from eighth generation descendants of one of the four A425 pair matings giving rise to $\mathrm{G}$. $\mathrm{H}$ showed a sex ratio at adult emergence of $30-40 \%$ females at $26 \times 2{ }^{\circ} \mathrm{C}$ during eight generations of further intermittent selection for $M P$ expression before investigation.

$W$ : A laboratory strain with an undistorted sex ratio, homozygous for the autosomal locus white pupa (wp), obtained from Dr Yoram Rössler, Ness Ziona, Israel.

SEIBERSDORF: A laboratory strain with an undistorted sex ratio, obtained from the International Atomic Energy Agency at Seibersdorf, Austria.

DC: A laboratory strain with an undistorted sex ratio, obtained from Dr Yoram Rössler, Ness Ziona, Israel.

\section{Rearing, temperature treatment and crossing}

Rearing was normally carried out at $26 \pm 2{ }^{\circ} \mathrm{C}$. The adults, fed on a mixture of sugar and hydrolysed yeast, were housed in clear plastic cages, with one side of fine mesh, through which the eggs were laid, falling into water. The larvae were reared on a carrot diet (Busch-Petersen and Wood, 1986), from which they jumped into sand to pupate. Puparia for experimental treatment were maintained in an incubator at $18 \pm 1.5^{\circ} \mathrm{C}$ for the first 5 days of pupal development, to include the critical period for the enhancement of $M P$ expression, determined by Wood and Shahjahan (1988). Crosses were made with 10 to 20 flies of each sex. $G$ flies for crossing were sampled from the $F_{2}$ progenies of pair matings in which the puparia had been kept for the first 5 days after pupation at $18^{\circ} \mathrm{C}$. $\mathrm{W}$ flies were taken directly from stock. Under the conditions of laboratory rearing described above, medfly males were ready to mate within 7 days of eclosion from the puparium.

\section{Electron microscope studies of spermiogenesis}

The preparation of testes for electron microscopy began with the dissection of 5-day adults in Ringer's solution. The testes were fixed in $2.5 \%(\mathrm{v} / \mathrm{v})$ gluteraldehyde in $0.1 \mathrm{M}$ sodium cacodylate buffer, $\mathrm{pH} 7.4$, for $2 \frac{1}{2} \mathrm{~h}$. They were then buffer-washed for $5 \mathrm{~min}$ and postfixed in $1 \%$ $(\mathrm{w} / \mathrm{v})$ osmium tetroxide in sodium cacodylate buffer for $1 \mathrm{~h}$, buffer-washed for $5 \mathrm{~min}$, dehydrated in alcohol and embedded in Spurr's resin. Ultrathin sections were stained in 2\% (w/v) uranyl acetate in $70 \%$ alcohol for $20 \mathrm{~min}$ and $0.3 \%$ lead citrate in $0.1 \mathrm{M}$ sodium hydroxide for $5 \mathrm{~min}$ before examination. Counts were made on normal and abnormal sperms per cyst.

\section{Results}

Reciprocal crosses and back-crosses between $\mathrm{W}$ and $\mathrm{G}$ In Table 1 each reciprocal cross and back-cross is based on three or four independent replicates, each one drawing its $G$ parent, or component, from a different

Table 1 Sex ratio observed in newly emerged adults from reciprocal crosses and back-crosses between $W$ and $G$ strains of the Mediterranean fruit fly $C$. capitata, based on three or four replicates in each case, except for $\mathrm{W} \times \mathrm{W}$, based on stock cage sampling

\begin{tabular}{|c|c|c|c|c|c|c|c|}
\hline \multirow[t]{2}{*}{ No. } & \multirow{2}{*}{$\begin{array}{c}\text { Cross } \\
\text { Female } \times \text { male }\end{array}$} & \multicolumn{5}{|c|}{ Sex ratio } & \multirow{2}{*}{$\begin{array}{c}\text { Replicate range } \\
\% \text { female }\end{array}$} \\
\hline & & Female & Male & $\%$ Female & $\chi_{1: 1}^{2}$ & $\mathrm{P}$ & \\
\hline 1 & $\mathrm{G} \times \mathrm{G}$ & 344 & 717 & 32.42 & 131.1 & $* * *$ & $28.6-36.9$ \\
\hline 2 & $\mathrm{~W} \times \mathrm{W}$ & 712 & 737 & 49.14 & 0.43 & NS & - \\
\hline 3 & $\mathrm{~W} \times \mathrm{G}$ & 363 & 553 & 39.63 & 39.4 & $* * *$ & $34.0-44.6$ \\
\hline 4 & $\mathrm{G} \times W$ & 475 & 476 & 49.95 & - & NS & $49.3-51.1$ \\
\hline 5 & $(W \times G) \times G$ & 410 & 628 & 39.49 & 45.8 & $* * *$ & $35.7-45.2$ \\
\hline 6 & $(W \times G) \times W$ & 361 & 360 & 50.04 & - & NS & $49.8-50.9$ \\
\hline 7 & $\mathrm{G} \times(\mathrm{W} \times \mathrm{G})$ & 542 & 602 & 47.38 & 5.6 & * & $40.9-55.6$ \\
\hline 8 & $\mathrm{~W} \times(\mathrm{W} \times \mathrm{G})$ & 359 & 444 & 44.71 & 9.0 & $* *$ & $40.4-48.4$ \\
\hline 9 & $(G \times W) \times G$ & 325 & 482 & 40.27 & 30.5 & $* * *$ & $35.3-43.9$ \\
\hline 10 & $(G \times W) \times W$ & 284 & 307 & 48.05 & - & NS & $46.4-50.3$ \\
\hline 11 & $\mathrm{~W} \times(\mathrm{G} \times \mathrm{W})$ & 290 & 332 & 46.62 & 3.1 & NS & $44.7-48.4$ \\
\hline 12 & $\mathrm{G} \times(\mathrm{G} \times W)$ & 621 & 597 & 50.98 & 0.8 & NS & $49.3-53.2$ \\
\hline 13 & $\mathrm{G} \times \mathrm{G}$ & 321 & 545 & 37.07 & 57.9 & $* * *$ & $33.6-38.5$ \\
\hline 14 & $\mathrm{~W} \times \mathrm{W}$ & 592 & 599 & 49.71 & - & NS & - \\
\hline
\end{tabular}

The replicates were independent although related, being drawn from F2 progenies of single pair matings of siblings. Crosses 1 and 2 were carried out before crosses 3-12, and crosses 13 and 14 afterwards.

NS, not significant.

${ }^{*} P<0.05 ;{ }^{* *} P<0.01 ;{ }^{* * *} P<0.001$. 
single pair $(G \times G) F_{2}$ progeny. It was necessary to sample from $F_{2}$ progenies (rather than $F_{1}$ ) in order to have enough flies to make the full range of crosses with 10 to 20 individuals of each sex. This meant that for the $G$ flies used in crosses, it was their grandparents that had been kept for the first 5 days after pupation at $18 \pm 1.5^{\circ} \mathrm{C}$. Each of the two $\mathrm{G} \times \mathrm{G}$ matings (carried out before and after the crosses) is based on four independent replicates. The two $\mathrm{W} \times \mathrm{W}$ matings are based on mass rearings in stock cages. Sex ratio was scored in newly emerged adults.

The sex ratio from $\mathrm{W} \times \mathrm{W}$ did not differ from parity $\left(\chi^{2}\right.$ test) in either sample tested (2 and 14 in Table 1), whereas in $G \times G$ there was a significant excess of males in both cases (1 and 13 in Table 1). The pattern of inheritance in the crosses was holandric in the sense that a G Ychromosome was involved in every instance of sex ratio distortion (matings 1, 3, 5, 7, 8, 9 and 13 in Table 1). The pattern was modified, however, when the G Y-chromosome was paired with a W X-chromosome, which showed a degree of resistance to the $G$ effect (matings 7 and 8), compared with when it was paired with its own $\mathrm{X}$-chromosome (matings 1, 3, 5, 9 and 13). The pattern is one of Y-chromosome meiotic advantage, that is, meiotic drive (Figure 1).
AAXX X AAXY

$* * *$

AAXY X AAXX : AAXY X AAXX

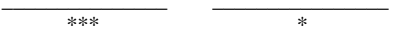

AAXY $x$ AAXX : AAXY $x$ AAXX

NS
AAXX $x$ AAXY

NS

AAXY X AAXX : AAXY X AAXX

$\overline{* * *} \overline{\mathrm{NS}}$

AAXY x AAXX : AAXY X AAXX

NS NS
Figure 1 The reciprocal crosses and back-crosses shown in Table 1 indicating the autosomal complement and sex chromosomes are as follows: $W$ males, AAXY; W females, AAXX; G males, AAXY; G females, AAXX. Values of probability $(p)$ of $\chi^{2}(1: 1)$ are as in Table 1.
The W strain was homozygous for the autosomal marker gene wp. Cross 8 in Table 1 represented a test of possible pseudolinkage between the Y-chromosome and the $w p^{+}$allele. (The optical examination made by Wood et al (1986) did not preclude minor exchanges.) The test cross was wp/wp $\mathrm{X}^{\mathrm{w}} / \mathrm{X}^{\mathrm{w}} \times \mathrm{wp} /+\mathrm{X}^{\mathrm{w}} / \mathrm{Y}^{\mathrm{G}}$, giving progeny as follows: wp/wp $X^{w} / X^{w}$ 181: $w p /+X^{w} /$ $X^{\mathrm{w}}$ 178: $w \mathrm{p} / \mathrm{wp} \mathrm{X}^{\mathrm{w}} / \mathrm{Y}^{\mathrm{G}}$ 210: $\mathrm{wp} /+\mathrm{X}^{\mathrm{w}} / \mathrm{Y}^{\mathrm{G}}$ 234. There was no evidence of pseudolinkage in this case.

Mortality during development in $G$ single pair $F_{1}$ families showing different degrees of sex ratio distortion

Sex ratio distortion in $G$ single pair $F_{1}$ families was sufficiently variable to raise the possibility of an influence from postzygotic mortality. This was investigated in a sample of 17 single pair families the parents of which were kept for the first 5 days after pupation at $18 \pm 1.5^{\circ} \mathrm{C}$. Eggs were collected from adults during the first 2 days of oviposition (mean number of eggs per pair $=127$, standard deviation $(S D)=63.3$ ). Mortality varied between families (eggs yielding first instar larvae 55.8-73.6\%; first instar larvae developing to pupae 22.6$60.2 \%$; eggs yielding adults $13.8-33.3 \%$ ). The frequency of females in families varied between 17.4 and $46.7 \%$, but no significant relationship could be found between sex ratio and mortality at any stage. This can be judged from values of correlation coefficient $(r)$, calculated on arc sine-transformed percentages between female frequency and the various values of yield. These were as follows: eggs yielding first instar larvae ( $\%$ hatch), $r=-0.039$; first instar larvae developing to pupae, $r=0.023$; and eggs yielding adults, $r=0.004$, all with 15 degrees of freedom and without significance. It was concluded that the sex ratio distortion was coming exclusively from a prezygotic effect, agreeing with the breeding evidence of Y-chromosome advantage.

\section{Sperm counts in individual spermatocysts}

All stages of spermiogenesis could be observed in 5-day adult testes, including the formation of testicular cysts,
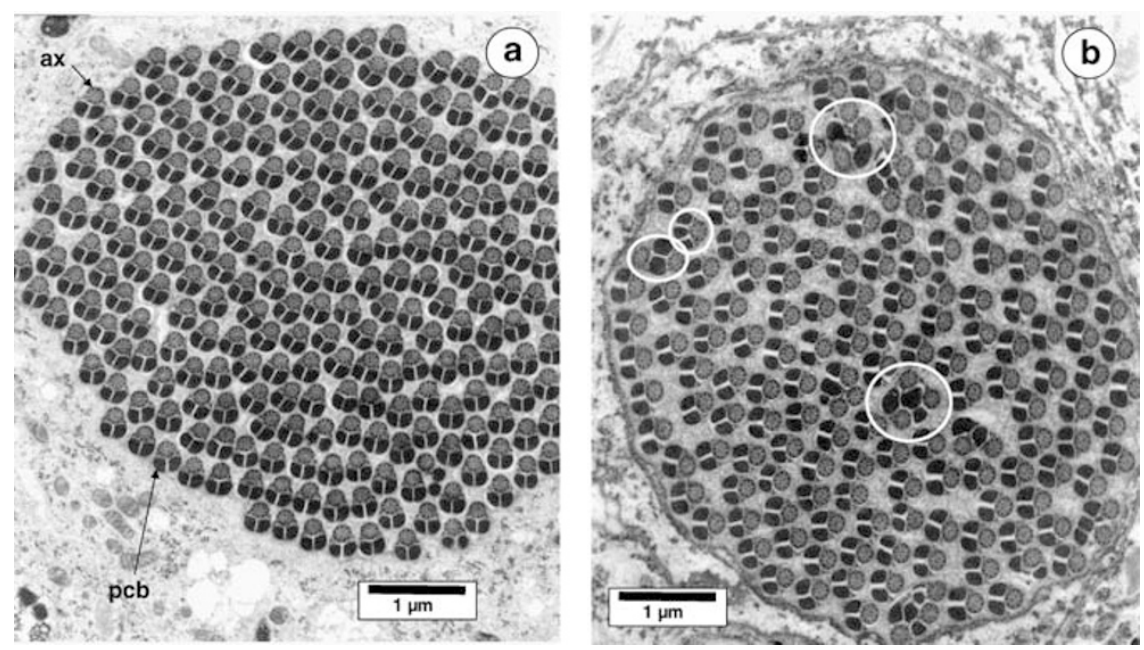

Figure 2 (a) Transverse section of sperm tails of the SEIBERSDORF strain of $C$. capitata within a single cyst. Each sperm is identical in showing a single axoneme (ax) and two paracrystalline bodies (pcb). (b) Transverse section of sperm tails of the MP strain of $C$. capitata within a single cyst. Some sperms show abnormal numbers of axonemes and/or PCB (four examples are circled), and the number of sperms is depleted below the normal range of 233-256 per cyst. 
a

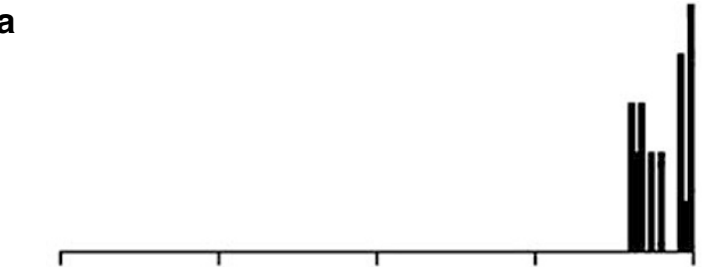

b

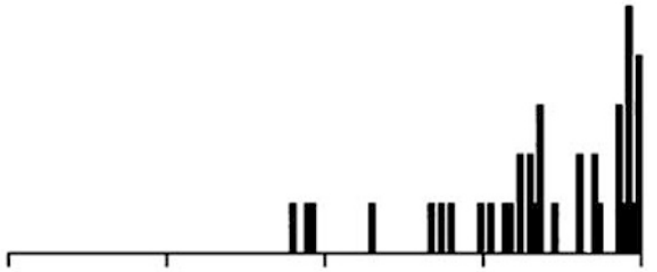

C

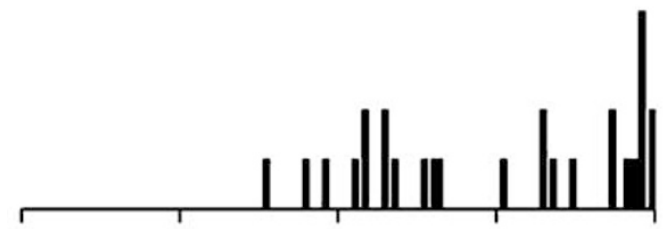

d

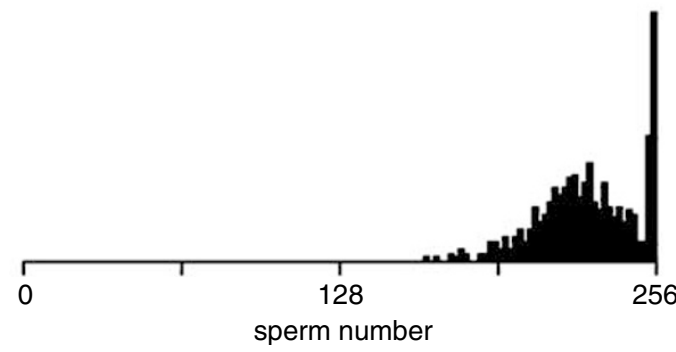

Figure 3 Frequency distributions of sperm number per cyst in testes of $C$. capitata. (a) Combined data on total sperms per cyst from two control strains SEIBERSDORF and DC. (b) Combined data on total sperms per cyst from two samples (MP-1, MP-2), from two different individuals, of the $M P$ strain, showing Y-chromosome meiotic drive. (c) Combined data on normal (nondeformed) sperms per cyst from the same MP samples as in (b). (d) A simulation based on random generation of values of sperm number for 500 cysts of the $M P$ strain, using the estimated value of $P=0.18$, this being the probability of reversion of an $\mathrm{MP}$ X-chromosome from potential sensitivity to Y-chromosome meiotic drive, to becoming insensitive, during one of the six generations before meiosis. the walls of which retained their integrity sufficiently long to permit accurate counts to be made of their sperm content. The maximum number of gametes within each cyst was 256. Sperm tails of regular form in a SEIBERSDORF cyst are shown in transverse section in Figure 2a. In this strain, and also in the other control strain DC, both with normal sex ratios, the number of sperms per cyst ranged from 233 to 256, (mean 244.4 \pm 9.8 in 11 cysts of SEIBERSDORF; 245.6 \pm 8.6 in 11 cysts of DC). In two samples from the $M P$ strain (taken from individual testes from two different individuals), the range of variation in sperms per cyst was substantially greater. The range was precisely the same in each case, although the means were different (122-256 in the 21 cysts of sample testis 1 , mean $209.7 \pm 34.1 ; 122-256$ in the 15 cysts of sample 2 , mean $225.7 \pm 43.1$ ). Some of the cysts in each $M P$ testis appeared normal, as in Figure 2a; in others, there was a reduction in sperm number, and disorientation and abnormality in some sperm, illustrated in Figure 2b.

The abnormalities evident in the tails of a proportion of $M P$ sperms took the form of extra axonemes and paracrystalline bodies (mitochondrial derivatives) (see four sperm tails circled in Figure 2b; other abnormal combinations of axonemes and paracrystalline bodies are also visible). Such abnormalities were never observed in either of the control strains. The frequency distribution of total sperm number per cyst in the two control strains (data combined) is shown in Figure 3a, compared with the two $M P$ samples combined in Figure $3 b$. Total data for the number of normal (ie nondeformed) sperms per cyst, in the two samples of $M P$, are shown in Figure 3c.

The mean numbers of normal sperms in the four sets of cysts are recorded in Table 2. In the two control strains, the numbers differ little or not at all from the total sperm number, but in the $M P$ sperms, abnormal ones represent a substantial proportion. The complement of normal sperms within each $M P$ cyst was, in some circumstances, greatly reduced, although in other cysts at the same stage of development, within the same testis, the number could be less reduced or similar to that in control strains. Counts of sperm number and deformed sperm number were made exclusively on sections cut at the posterior of the testis where the cysts were mature. The frequency of deformed sperms was assessed only in a subset of cysts

Table 2 Two samples of MP (MP-1, MP-2) compared with two control strains (SEIBERSDORF, DC) for mean number of sperms per cyst, mean number of sperms with nondeformed tails per cyst, number of sperms missing per cyst (out of a maximum of 256)

\begin{tabular}{|c|c|c|c|}
\hline Strain/sample & $\begin{array}{c}\text { Mean number of sperms/cyst } \\
\pm S D(\mathrm{~N})\end{array}$ & $\begin{array}{c}\text { Mean number of non-deformed sperms/cyst } \\
\pm S D(\mathrm{~N})\end{array}$ & $\begin{array}{c}\text { Mean number of sperms missing from } 256 \\
\pm S D(\mathrm{~N})\end{array}$ \\
\hline $\begin{array}{l}D C \\
\text { Subset }^{\mathrm{a}}\end{array}$ & $\begin{array}{l}245.6 \pm 8.6(11) \\
238.3 \pm 4.6(6)\end{array}$ & $234.8 \pm 7.0(6)$ & $10.5 \pm 8.6$ \\
\hline $\begin{array}{r}\text { SEIBERS } \\
\text { Subset }\end{array}$ & $\begin{array}{l}244.4 \pm 9.8(11) \\
246.4 \pm 9.4(7)\end{array}$ & $246.4 \pm 9.4(7)$ & $11.6 \pm 9.8(11)$ \\
\hline $\begin{array}{l}M P-1 \\
\text { Subset }\end{array}$ & $\begin{array}{l}209.7 \pm 34.1(21) \\
209.1 \pm 35.0(15)\end{array}$ & $185.7 \pm 45.6$ & $46.3 \pm 34.1$ \\
\hline $\begin{array}{l}\text { MP-2 } \\
\text { Subset }\end{array}$ & $\begin{array}{l}225.7 \pm 43.1(15) \\
220.4 \pm 47.9(13)\end{array}$ & $209.0 \pm 54.3(13)$ & $30.3 \pm 42.1$ (15) \\
\hline
\end{tabular}

$\mathrm{MP}$, male producer; SD, standard deviation.

Standard deviations are given, with sample numbers in brackets.

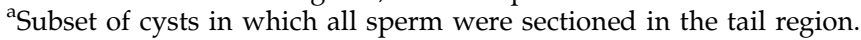


in which all sperms had been sectioned in the tail region (Table 2). Normal sperms numbered on average $185.7 \pm 45.6, N=15$, range $99-253)$ in $M P$ testis 1 , and $209.0 \pm 54.3, N=13$, range 135-256) in $M P$ testis 2 . Sperms with a deformed structure numbered $23.5 \pm 21.3$ in testis 1 , and $11.4 \pm 20.3$ in testis 2 . The sample numbers $(N)$ are smaller in the subsets, compared with total samples, because they exclude cysts containing sperms sectioned through the head. The numbers of sperms missing out of 256, in the four sets, are also recorded in Table 2. In the two control strains, the mean loss of 10.5 or 11.6 sperms per cyst was not associated with an effect on sex ratio.

Assuming that the missing and deformed sperms are those that would have been X-bearing, it is possible to assess the putative sex ratio from the eggs fertilised by the content of each cyst. Data from both testes are combined in this calculation. The frequencies of deformed sperm in the two samples $(23.5 \pm 21.3 \%(N=15)$ in $M P$ sample 1 and $11.4 \pm 20.3 \%(N=13)$ in sample 2$)$ were not significantly different $(P=0.14)$. Considerable variation was observed between cysts, three of which were expected to give no females at all (ie $50 \%$ or more sperms were missing), and others to give different degrees of distortion, so that the overall putative sex ratio comes out at $34.4 \%$ females $(S D=17.1, N=39)$. This value compares reasonably closely with the sex ratio actually observed in the progeny of male sibs, $36.7 \%$ females $(N=804)$. It is to be noted that there were some cysts containing fewer than 128 normal sperms, indicating a depletion of some male-determining sperms.

\section{A theory to explain the variation in sperm number per cyst in an MP strain}

The expression of $M P$ varies widely among cysts of the same individual. This variation cannot be genetic sensu stricto since each cyst has been derived from a single stem cell (primary spermatogonium) with the same genotype. Each stem cell destined to produce an individual cyst divides to form its independent cell lineage, yielding 2, 4, 8, 16, 32 and 64 mitotic products before meiosis increases the number to 256. In some cysts, $M P$ is apparently expressed to a maximum $(50 \%$ or fewer normal sperms), whereas in neighbouring cysts, it may show no expression (zero loss), or some intermediate condition. The variation cannot be attributed to the age of cysts or their position within the testis. All of them are at a mature stage in 5-day-old adults, in sections cut at a corresponding transverse plain from the posterior end of the testis. Yet, the evidence points to extreme variation between neighbouring cysts in the effect of $Y$ chromosome meiotic drive.

That the process we have attributed to Y-chromosome meiotic drive is initiated in some way at meiosis is supported by the period of temperature sensitivity identified by Wood and Shahjahan (1988). It is evident, however, that the potential for variability among cysts must arise earlier than meiosis, not later than during the six mitotic divisions before meiosis as the cysts are created. We are therefore led to conclude that during the six generations to produce 64 products derived from a single original stem cell, there is the opportunity for a proportion of these products to change in a manner yet to be explained. The change would be either towards or away from potential $X$ sensitivity to the action of the driving Y-chromosome during the process of meiosis that follows. It seems to make better evolutionary sense for the change to be away from sensitivity (a suppression of sensitivity), but the principle would apply equally in the opposite direction. It could also operate if the change concerned effectiveness of $\mathrm{Y}$ drive, rather than $\mathrm{X}$ sensitivity. In the case of $X$ sensitivity, the factor responsible is not necessarily on the $\mathrm{X}$-chromosome.

Considering the six mitotic divisions, it is possible to generate a distribution expressing variation in $X$ sensitivity by making two assumptions. The first is that there exists a certain probability of a change in X-chromosome sensitivity occurring at each of the mitotic divisions in the formation of a cyst from its stem cell. The second is that once such a change has occurred, it is passed to all descendants of that cell. Starting from the stem cell there is a probability $p(=1-q)$ of conversion from sensitive to insensitive in each of the two daughter cells. The next cell generation inherits from the parental cell a frequency $p$ converted, to which is added a fraction $p$ of the 1-p unconverted. The expected reverted fraction is now $p+p(1-p)=1-q^{2}$. The inherited part of this quantity contributes twice as much to the variance as the newly reverted fraction, so that the expected binomial variance $q^{2}\left(1-q^{2}\right) / 4$ is increased by a factor $(2-p q) /\left(1-q^{2}\right)$. Continuing this argument over six cell generations, the final frequency converted, $r$, is $1-q^{6}$. Reduction division then results in up to 256 gametes, with no further conversion occurring. The binomial variance of $q^{6}\left(1-q^{6}\right) /$ 64 is now increased by a factor representing the contributions from each previous cell generation, which is a summation approximating to $64(1-q) /\left((2-q)\left(1-q^{6}\right)\right)$.

From the data, the mean $M P$ sperm count per cyst is 217.1, with some cysts reaching 256. Assuming that $128 \mathrm{Y}$ sperm survive, the frequency of reverted $X$-bearing sperm is $r=(217.1-128) / 128$, or 0.696 . Consequently, $p$, the probability of reversion at each cell division, is 0.18 . A simulation based on random generation of values for 500 cysts using this estimate is shown in Figure $2 \mathrm{~d}$. The resemblance with the experimental data (Figure $2 \mathrm{~b}$ and c) is only partial. The model makes no allowance for loss of sperm (both $X$ and $Y$ ) owing to other causes, as observed in both control strains (Figure 2a). This would move the distribution in Figure $2 \mathrm{~d}$ to the left. In the model, we have assumed conversion from sensitive to insensitive to be a one-step process. If the process occurred in increments, over several cell generations, sensitivity would be lost more gradually, and the distribution would be extended further to the left. The point of the model, however, is not to guess at parameters that might produce a closer simulation but simply to examine how variation between cysts of identical genotype could arise, given the possibility of some process by which potential $\mathrm{Y}$ advantage at meiosis might be reduced.

\section{Discussion}

The male-producing factor $M P$ in the medfly, identified in this study by its effect on sperm development as well as primary sex ratio, is inherited only through the male sex. The father-to-son pattern of inheritance, coupled with the fact that sex ratio distortion is independent of postzygotic mortality, points to the mechanism being one 
of Y-chromosome advantage at meiosis (Y-chromosome meiotic drive). As such, MP must be closely associated with the male-determining factor $M$, and cause $M$ to drive against its supposed X-chromosome allele. The advantage of the $M P$ Y-chromosome is demonstrably variable, indicating variability in the genome within which it is acting. Sensitivity is inherited through either sex and is unstable, showing a tendency to decline between generations when selection is relaxed. The MP factor thus shows properties similar to those characterising the Y-linked $D$ (distorter) gene (also referred to as $\mathrm{MD}$ ) in the mosquito Aedes aegypti (Hickey and Craig, 1966; Wood, 1976; Wood and Newton, 1991).

In $A$. aegypti, the driving Y-chromosome acts by causing the $\mathrm{X}$-chromosome to fragment at meiosis (Newton et al, 1976), whereas two major X-linked loci control $X$ sensitivity (Wood and Ouda, 1987). An exploratory examination of meiosis in feulgen-stained testicular tissue of $M P$ failed to reveal any evidence of fragmentation, or any other obvious sign of chromosomal abnormality (Wood et al, 1986). Instead, examination of spermiogenesis by electron microscopy revealed an unusually high degree of spermiogenic mortality associated with the sex ratio distortion, showing that the effect on sex ratio was truly prezygotic. Spermiogenesis associated with MP showed features not observed in the SEIBERSDORF or DC control strains. The differences were (1) the loss of a proportion of sperm, (2) the presence of abnormal arrangements of organelles in some of the surviving sperm. The average number of missing and deformed sperms in $M P$ was roughly consistent with the average depression in female recovery among progenies of siblings.

The simplest explanation for the origin of the $M P$ factor is from exposure to the irradiation imposed on the single male from which the strain was derived, although it could be a natural variant. An effect of irradiation on the $\mathrm{X}$-chromosome is precluded by three generations of out-crossing of the irradiated male to nonirradiated females (Wood et al, 1986). In two other studies of the medfly, distorted sex ratios have been observed without irradiation: sex ratio-distorting genes at two separate autosomal loci were reported by Malacrida et al (1987), whereas Saul and McCombs (1993) found stable sex ratio distortion associated with a chromosome rearrangement. Cytological examination revealed no evidence of a chromosome anomaly in the present case (Wood et al, 1986), but something invisible microscopically could not be excluded. For this reason, we examined our data for evidence of pseudolinkage that might be associated with a chromosome rearrangement. There is no $\mathrm{Y}$ linkage with the autosomal marker $w p$, or with the autosomal genetic basis of malathion resistance, reported earlier (Wood et al, 1986). A chromosome rearrangement involving the Y-chromosome might have revealed itself by postzygotic differential mortality of the sexes, but that has been excluded experimentally.

The MP condition is sensitive to temperature during spermatogenesis. The proportion of males in the progeny was found to increase significantly when temperature at the onset of meiosis was lowered from 26 to $18^{\circ} \mathrm{C}$ (Wood et al, 1986; Wood and Shahjahan, 1988), although temperature at other stages of development was without effect (RM Shahjahan, unpublished data). Degeneration of the X-chromosome appears to start at the first meiotic division. A parallel example of temperature sensitivity was reported in a sex ratio mutation in Drosophila simulans (Ramamurthy et al, 1980). In this case, the bias was towards females, as a result of $\mathrm{X}$-chromosome meiotic drive. The degeneration of some of the sperm in a bundle (ie cyst) was characteristic. Montchamp-Moreau and Joly (1997) showed that the degree of spermiogenic failure was correlated with the bias towards females in their progeny.

In seeking an explanation for the heterogeneity in spermiogenic failure among cysts in the present study, we suggest that certain X-bearing gametes, but not others, become affected, even though all are of the same genotype in any one male. The explanation that has been proposed links it to variation in X-chromosome sensitivity to $M P$, arising by random suppression of the genetic basis of sensitivity (either sex linked or autosomal) during the six mitotic divisions in the origin of the cyst from its stem cell (primary spermatogonia), before meiosis. To account for the wide variation among cysts within the same testis, we propose that X-chromosome insensitivity arises randomly during these six divisions. We refer to this as conversion. The evidence suggests conversion of approximately 0.18 per cell generation, much more frequent than a normal mutation. The wide variability among cysts in degrees of sperm depletion can then be explained in terms of the mitotic generation in which conversion occurred.

Variation in gene expression between cysts within the same testis is not unique. A similar phenomenon has been reported with respect to caspase activity affecting sperm individualisation in Drosophila melanogaster (Huh et al, 2004). Testes from flies mutant for several caspase pathways contained cysts in which there was a complete failure of individualisation, whereas other cysts in the same testis carried out the process apparently normally. The authors present evidence consistent with the idea that the stochastic nature of this variability may reflect a requirement for a threshold level of caspase activity in order for a cyst to initiate individualisation. In $M P$ medfly spermiogenesis, the mechanism has to result in continuous variation, the individualisation of some sperms but not others occurring at varying frequencies per cyst.

Whenever sex ratio-distorting genes are found in pest insects, it is natural to think about their possible role in controlling that pest. In its present form, $M P$ has too slight an effect and is too unstable to be of value in this respect. The question is whether it or something similar can be improved. Strains of $A$. aegypt $i$ with sex ratios less than $6 \%$ females were produced by $M D$ meiotic drive, but could not easily be maintained because of low fertility (Pearson and Wood, 1980), whereas a sex ratio around $30 \%$ could be kept stable (Owusu-Daaku et al, 1997) but had no practical application.

For Y-chromosome meiotic drive to be valuable for pest control, it needs to be extreme enough to produce males alone. At the same time, the system must in some way be brought under conditional control, so as to generate females for breeding. Experience with dietcontrolled lethality in D. melanogaster (Thomas et al, 2000) shows that dietary control might be the answer, easier to manage than temperature conditioning in mass production. Once a conditional system is in place, the scope would be widened for further selection without the 
problem of infertility. First, however, it is necessary to determine the molecular basis of Y-chromosome meiotic drive.

\section{Acknowledgements}

The breeding work was conducted under FAO/IAEA Research Contract No. 2488/TC. RMS was supported by a Commonwealth Scholarship. The cytological studies by PR were funded by the International Atomic Agency, The Committee of Vice Chancellors and Principals of British Universities (ORS Scheme), Manchester University bursaries, and the Guatemala Medfly Control Program. We thank Dr Paul Higgs for his valuable input into our early discussion on how an error rate in the formation of a cyst from its stem cell might explain the heterogeneity in $M P$ expression observed in different cysts within the same testis. We thank Professor Christopher F Curtis and Dr James Visanji for helpful comments on an earlier draft of this paper. Two anonymous referees gave valuable advice on getting the final version into shape.

\section{References}

Anwar M, Chambers DL, Ohinata K, Kobayashi RM (1971) Radiation sterilization of the Mediterranean fruit fly (Diptera: Tephitidae); comparison of spermatogenesis in flies treated as a pupae or adults. Ann Entomol Soc Am 64: 627-633.

Busch-Petersen E, Wood RJ (1986). The isolation and inheritance of dieldrin resistance in the Mediterranean fruit-fly, Ceratitis capitata (Wiedemann) (Diptera, Tephritidae). Bull Entomol Res 76: 567-581.

Causse R (1972). Ontogenèse des cellules reproductrices chez Ceratitis capitata Wiedemann (Diptera: Trypetidae). Ann Zool Ecol Anim 4: 35-53.

Hickey WA, Craig GB (1966). Distortion of sex ratio in populations of Aedes aegypti. Can J Genet Cytol 8: 260-278.

Huh JR, Vernooy SY, Yu H, Yan N, Shi Y, Guo M et al (2004). Multiple apoptotic caspase cascades are required in nonapoptotic roles for Drosophila spermatid individualisation. PloS Biol ( http://biology.plosjournals.org) 2: 43-53.

Malacrida A, Gasperi G, Milani R (1987). Genome organization of Ceratitis capitata: linkage group and evidence for sex ratio distorters. In: Economopoulos A (ed) Fruit Flies. Elsevier Science Publications: Amsterdam. pp 169-174.

Montchamp-Moreau C, Joly D (1997). Abnormal spermiogenesis is associated with the X-linked sex-ratio trait in Drosophila simulans. Heredity 79: 24-30.
Newton ME, Wood RJ, Southern DI (1976). A cytogenetic analysis of meiotic drive in the mosquito Aedes aegypti. Genetica 46: 297-318.

Owusu-Daaku KO, Wood RJ, Butler RD (1997). Selected lines of Aedes aegypti with persistently distorted sex ratios. Heredity 79: 388-393.

Pane A, De Simone A, Saccone G, Polito CL (2005). Evolutionary conservation of Ceratitis capitata transformer gene function. Genetics 171: 615-624.

Pane A, Salvemini M, Delli Bovi P, Polito C, Saccone G (2002). The transformer gene in Ceratitis capitata provides a genetic basis for selecting and remembering the sexual fate. Development 129: 3715-3725.

Pearson AM, Wood RJ (1980). Combining the meiotic drive gene $\mathrm{D}$ and the translocation $\mathrm{T}_{1}$ in the mosquito Aedes aegypti (L.). 1. Sex ratio distortion and fertility. Genetica 51: 203-210.

Phillips DM (1970). Insect sperm: their structure and morphogenesis. J Cell Biol 44: 234-277.

Ramamurthy G, Alfert M, Stern C (1980). Ultrastructural studies on spermatogenesis in a sex ratio mutant strain of Drosophila simulans. Am J Anat 157: 205-219.

Saul SH, McCombs SD (1993). Stable sex ratio distortion in the Mediterranean fruit fly Ceratitis capitata (Diptera: Tephritidae) associated with a chromosomal rearrangement. Ann Entomol Soc Am 86: 203-206.

Thomas DD, Donnelly CA, Wood RJ, Alphey LS (2000). Insect population control using a dominant, repressible, lethal genetic system. Science 287: 2474-2476.

Willhoeft U, Franz G (1996). Identification of the sex determining region of the Ceratitis capitata $\mathrm{Y}$ chromosome by deletion mapping. Genetics 144: 737-745.

Wood RJ (1976). Between-family variation in sex ratio in the Trinidad (T-30) strain of Aedes aegypti (L.) indicating differences in sensitivity to the meiotic drive gene $M^{D}$. Genetica 46: 345-361.

Wood RJ, Newton ME (1991). Sex ratio distortion caused by meiotic drive in mosquitoes. Am Nat 137: 379-391.

Wood RJ, Ouda NA (1987). The genetic basis of resistance and sensitivity to the meiotic drive gene $D$ in the mosquito Aedes aegypti L. Genetica 72: 69-79.

Wood RJ, Saaid SS, Shahjahan RM, Southern DI (1986). Excess male production in lines of the Mediterranean fruit fly Ceratitis capitata (Wied.) isolated after X-irradiation followed by outcrossing. In: Economopoulos A (ed) Fruit Flies. Elsevier Science Publications: Amsterdam. pp 179-188.

Wood RJ, Shahjahan RM (1988). Temperature dependent sex ratio distortion after X-irradiation of medfly males. In: Modern Insect Control: Nuclear Techniques and Biotechnology. International Atomic Energy Agency: Vienna. pp 195-203. 